\section{Chrischta Ganz}

\section{Basische Ernährung}

Für den Säure-Basen-Haushalt ist entscheidend, ob ein Nahrungsmittel im Gewebestoffwechsel sauer oder basisch wirkt (und nicht, ob es sauer schmeckt oder nicht). Da saure Valenzen nicht im Blut verbleiben dürfen, lagern sie sich bei mangelnder Ausscheidung in der extrazellulären Matrix (Interstitium, Pischinger Raum) ab. Deshalb werden in der basischen Ernährung Nahrungsmittel bevorzugt, die basisch verstoffwechselt werden (Gemüse, Kartoffeln, Früchte, Kräuter).

\section{Vollwerternährung}

Vollwerternährung besteht aus pflanzlichen Lebensmitteln wie Vollgetreide, Gemüse und Obst sowie Milch und Milchprodukten. Fleisch und Fisch spielen eine untergeordnete Rolle. Übertriebene Verarbeitung der Lebensmittel sowie Zusatz- und Konservierungsstoffe werden möglichst vermieden. Bevorzugt werden Lebensmittel aus kontrolliert ökologischem Anbau, möglichst saisongerecht, aus heimischen Anbaugebieten sowie sozialverträglich produziert.

Wichtige Begründer der Vollwerternährung sind der Arzt und Naturheilkundler Maximilian BircherBenner (1867-1939), der Arzt und Ernährungsfachmann Prof. Werner Kollath (1892-1970) sowie der Arzt Max Otto Bruker (1909-2001).

\section{Vegetarismus}

Gründe für eine vegetarische Ernährung sind ökologische Kriterien (für $1 \mathrm{~kg}$ Fleisch werden $10 \mathrm{~kg}$ Getreide verfüttert), der Protest gegen die nicht tiergerechten Zustände in der Tierhaltung sowie gesundheit-

\title{
Besondere Ernährungsformen
}

Nachdem in der letzten Ausgabe der Schweizerischen Zeitschrift für GanzheitsMEDIZIN die gesunde Ernährung in ihren Grundzügen skizziert wurde, werden hier verschiedene Ernährungsformen kurz vorgestellt (ohne Anspruch auf Vollständigkeit) (Abb. 1). Aus gesundheitlicher Sicht sind nicht alle empfehlenswert, doch ist es im Praxisalltag von Vorteil, einen Überblick über die wichtigsten Ernährungsformen zu haben.

liche Aspekte. Die bekanntesten Formen des Vegetarismus sind:

- Ovo-Lacto-Vegetarismus: Gemieden werden Lebensmittel, die aus dem Körper getöteter Tiere hergestellt werden (Wurst, Geflügel, Fisch, Gelatine, Schlachtfette). Eier, Milch und Milchprodukte hingegen werden verzehrt.

- Lacto-Vegetarismus: Vegetarische Kost mit zusätzlichem Verzicht auf Eier. Milch und Milchprodukte werden jedoch verwendet.

- Vegane Ernährung: Tierische Nahrung (auch alle Milchprodukte und Honig) wird vermieden, meist generell alle tierischen Produkte (Lederprodukte, Arzneien tierischen Ursprungs usw.).

- Rohkost: Pflanzliche Rohkost, ausschliesslich aus nicht erhitzten pflanzlichen Lebensmitteln.

- Frutarier-Ernährung: Es wird nur pflanzliche Nahrung, bei der die Pflanze selbst nicht getötet wird, gegessen; z.B. Äpfel, Nüsse und andere Baumfrüchte, aber kein Getreide, Wurzelgemüse oder Kopfsalate.

- Pudding-Vegetarismus: Umgangssprachliche Bezeichnung für eine «normale» Mischkost, bei der das Fleisch weggelassen wird.

- Fisch-Vegetarismus: Es wird auf Fleisch verzichtet, Fisch wird jedoch gegessen. Fisch-Vegetarier werden von «echten» Vegetariern als Pseudovegetarier bezeichnet.

\section{Makrobiotik}

Der Begriff Makrobiotik bezeichnet eine vegane Ernährung, die sich an hippokratischen Richtlinien orientiert und als deutscher Begriff von C.W. Hufeland (1762-1836) eingeführt wurde. Heute wird der Begriff vor allem als Bezeichnung der Ernährungslehre nach Sagen Ishizuka (1850-1909) verwendet [1], die durch Nyoichi Oshawa (1893-1966) populär geworden ist.

\section{Fasten und Entgiftung}

Ziel des Fastens ist eine Reinigung und Umstimmung des Körpers, da der Organismus beim Fasten entwässert, seine Fettspeicher abbaut und Toxine (insbesondere belastende Stoffwechselendprodukte) ausscheidet. Dabei setzt sich der Körper auch aktiv mit chronischen Krankheitsprozessen auseinander, was Regenerationsprozesse in Gang setzt.

Beim Fasten ist neben der körperlichen Ebene auch die seelischgeistige Ebene angesprochen. Auch können dabei schlechte Gewohnheiten abgelegt werden (Rauchen, Kaffee- und Alkoholgenuss). Es gibt viele verschiedene Arten von Fastenkuren (Tee-, Molke-, Saft-Fasten nach Buchinger-Lützner, normale Ernährung mit Verzicht auf Fleisch, Alkohol und Kaffee usw.). Die Art des Fastens, die Dauer sowie die Begleitmassnahmen (Darm!) sind individuell anzupassen.

\section{KARGER}

Fax +497614520714 Information@Karger.com Information@Kar.
(๑) 2013 S. Karger GmbH, Freiburg 


\section{F.X.-Mayr-Kur}

F.X. Mayr (1875-1965) postulierte, dass die meisten gesundheitlichen Störungen durch eine schlechte Verdauung bzw. einen kranken Darm ausgelöst werden. Am bekanntesten sind seine Milch-Semmel-Diät und Mayrs Betonung des guten Kauens: Er empfahl, jeden Bissen 50-mal zu kauen.

\section{Schroth-Ernährung}

Johann Schroth (1798-1856) empfiehlt eine eiweiss-, fett- und kochsalzfreie bzw. -arme Kohlenhydrat-Ernährung sowie Durst- und Trinktage im Wechsel, ergänzt mit physikalischen Therapien [2].

\section{Ernährung nach den 4 Elementen}

Aufbauend auf der Traditionellen Europäischen Naturheilkunde (TEN) werden die Lebensmittel und Zubereitungsarten nach ihren Qualitäten den Elementen Wasser (feucht und kalt), Luft (feucht und warm), Feuer (trocken und warm) und Erde (trocken und kalt) zugeordnet. Diese werden entsprechend der Konstitution sowie der aktuellen Lebenssituation ausgewählt.

Ernährung nach Hildegard von Bingen In ihrem Werk «Physica» teilt Hildegard von Bingen (1098-1179) die Nahrungsmittel gemäss der $\mathrm{Hu}$ morallehre den Elementarqualitäten warm, kalt, trocken und feucht zu. Masshalten ist ihre übergeordnete Empfehlung: «Die Seele liebt in allen Dingen das diskrete Mass. Wann auch immer der Körper des Menschen ohne Diskretion isst und trinkt oder etwas anderes dieser Art verrichtet, werden die Kräfte der Seele verletzt. In allen Dingen soll sich der Mensch selbst das rechte Mass auferlegen» [3].

\section{Ernährung nach den 5 Elementen}

Die Ernährung nach den 5 Elementen gründet auf der Lehre der Traditionellen Chinesischen Medizin (TCM)

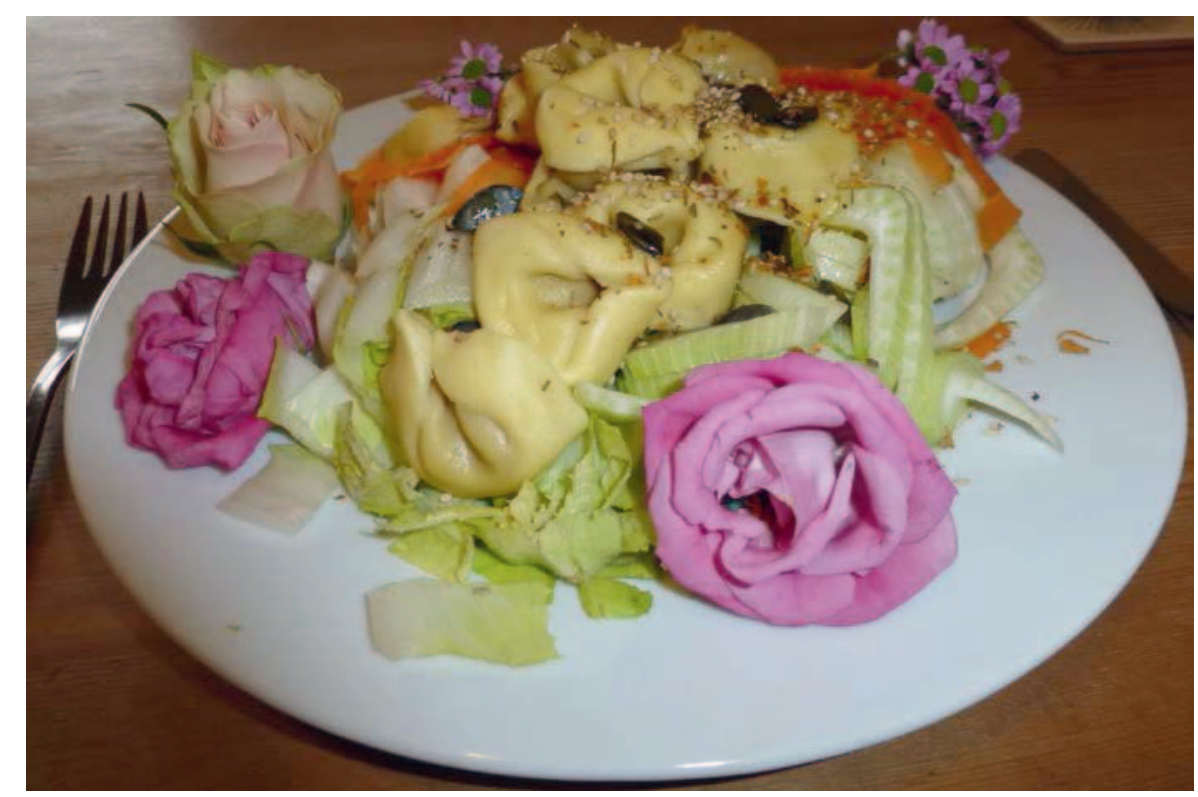

Abb. 1. Auch das Auge isst mit.

und ordnet alle Nahrungsmittel den Elementen Erde (süss), Metall (scharf), Wasser (salzig), Holz (sauer) und Feuer (bitter) zu. Wichtig bei der Zubereitung ist die Reihenfolge der zugefügten Lebensmittel gemäss dem Kreislauf der Wandlungsphasen [4].

\section{Ayurvedische Ernährung}

Die ayurvedische Ernährung richtet sich nach den Doshas Kapha (Erde/ Wasser), Pitta (Feuer) und Vata (Luft/Äther). Meist ist sie im konkreten Fall eine eher fleischarme Mischkost.

\section{Anthroposophische Ernährung}

Aus der Sicht Rudolf Steiners (18611925) wird der Wert der Nahrungsmittel durch ihre elementaren Bildekräfte (gestaltende, universelle Kräfte) bestimmt. Um die hohe Lebensmittelqualität $\mathrm{zu}$ gewährleisten und irdische und kosmische Kräfte $\mathrm{zu}$ harmonisieren, entwickelte er die anthroposophische Landwirtschaft, auch biologisch-dynamische Landwirtschaft genannt.

Gemäss Steiner wirken Wurzeln auf den Nerven-Sinnes-Pol (Nerven/ Sinnesorgane), Blätter und Stängel auf das rhythmische System (Herz/ Lunge), Früchte und Blüten auf den Stoffwechsel-Pol. Eine gezielte Nahrungsauswahl hält das Gleichgewicht dieser drei Pole aufrecht bzw. stellt es wieder her. Zusätzlich werden die Getreidearten Weizen, Reis, Gerste, Hirse, Roggen, Hafer und Mais gemäss astrologischen Gedankengutes den einzelnen Wochentagen zugeordnet.

Die anthroposophische Ernährung ist eine weitgehend ovo-lacto-vegetabile Kost mit gelegentlichem Fleischkonsum [1].

\section{Haysche Trennkost}

In einer Mahlzeit werden entweder protein- oder kohlenhydrathaltige Lebensmittel gegessen (jeweils in Kombination mit sogenannten «neutralen» Nahrungsmitteln). Es wird ausserdem zwischen basen- und säurebildenden Lebensmitteln unterschieden. Die Trennkost geht auf den amerikanischen Arzt Howard Hay (1866-1940) zurück. Später entstand aus der Hayschen Trennkost die Fit-for-Life-Diät (nach Harvey und Marilyn Diamond, 1985) sowie die Vitalstoffdiät der Annabelle. 


\section{Ernährung nach Blutgruppen}

Die Blutgruppendiät des amerikanischen Naturheilpraktikers Peter D’Adamo $\left({ }^{\star} 1956\right)$ geht davon aus, dass der Stoffwechsel je nach Blutgruppe anders arbeitet. Seine Theorie begründet er mit Lektinen aus der Nahrung, die bestimmten Membranproteinen der Erythrozyten ähneln $[5]$.

\section{Metabolic Typing/Ernährung nach Stoffwechseltypen}

Die Ernährung nach Stoffwechseltypen geht auf William R. Kelley und Bill Willcott aus Texas in den 1930er-Jahren zurück. Sie kombinierten die Blutgruppendiät mit typologischen Kriterien des Verbrennungssystems (Schnell-, Langsam- und Gleichmässig-Verbrenner), des Nervensystems (Sympathikus-, Parasympathikus- und ausgewogener Nerventyp) sowie des Drüsensystems (Hypophysen-, Schilddrüsen-, Nebennieren- und Gonadentyp) [6].

\section{Functional Food}

Als erstes Functional-Food-Produkt gilt die gute alte Ovomaltine. Heute hat Functional Food oft mehr mit Marketingstrategien als mit Gesundheit zu tun. Man denke an PommesChips, die mit ihrem Gehalt an Magnesium werben, oder an überzuckerte Kinderriegel mit der Extraportion Milch.

\section{Slow Food}

Slow Food ist als kulinarische Gegenbewegung zum Fast Food von Carlo Petrini $\left({ }^{*} 1949\right)$ aus Italien gegründet worden, angeblich als Protestaktion gegen die Eröffnung einer McDonald's-Filiale in Rom. Hauptanliegen von Slow Food ist ein genussvolles, gemütliches und bewusstes Essen, gemeinsam an einem grossen Tisch.
Ernährung nach Johannes F. Coy

Die Diät nach Dr. Johannes Coy $\left.{ }^{*} 1963\right)$ ist in der alternativen Krebstherapie recht bekannt. Nach Coy sind die Krebszellen durch die Vergärung von Glucose zu Milchsäure über den TKTL1(Transketolase-like1)-Stoffwechsel in der Lage, sich gegen Immunprozesse zu schützen. Um in den Stoffwechsel der Zellen einzugreifen, schlägt er eine extrem kohlenhydratarme Ernährung mit hohem Anteil an hochwertigen Ölen, Proteinen, Ballaststoffen und sekundären Pflanzenstoffen sowie eine sanfte Entsäuerung vor [7].

\section{Bedarfsorientierte Ernährung}

Der deutsche Heilpraktiker Heinrich Tönnies (1936-2006) versteht seine Methode als Synthese aus wissenschaftlichen Erkenntnissen und praktischer Erfahrung. Unter bedarfsorientiert versteht er eine individuelle Ernährung, die zwischen anregenden und beruhigenden Lebensmitteln unterscheidet und dem Tagesrhythmus, der Jahreszeit und den Lebensumständen angepasst ist.

\section{Schnitzer-Kost}

Der Zahnarzt Johann Georg Schnitzer $\left.{ }^{*}{ }^{*} 1930\right)$ unterscheidet die SchnitzerIntensivkost (eine Rohkostdiät aus Getreide, Nüssen, Hülsenfrüchten, Wurzelgemüse und grünen Salaten) und die Schnitzer-Normalkost (eine ovo-lacto-vegetarische Ernährung). Er begründet seine Ernährungsempfehlungen mit dem menschlichen Gebiss.

\section{Diät nach glykämischem Index}

Es werden Nahrungsmittel mit tiefem glykämischem Index (GI) bevorzugt. Damit wird eine längere Sättigung erreicht und die Insulinausschüttung reduziert.
Bei der Glyx-Diät nach Marion Grillparzer $\left({ }^{*} 1961\right)$ werden Lebensmittel mit einem hohen glykämischen Index konsequent gemieden. Zu Fettgehalt und Menge an Kohlenhydraten wird keine Empfehlung gemacht.

\section{Low-Carb-Diät}

Die Menge an Kohlenhydraten wird zugunsten von Proteinen und Fetten eingeschränkt. Die Idee einer eingeschränkten Kohlenhydratzufuhr soll bis ins 19. Jahrhundert zurückgehen.

\section{Atkins-Diät}

Bei der Diät nach Robert Atkins (1930-2003) sind als Energielieferanten nur Fett und Eiweisse erlaubt. Im Gegensatz zur Low-Carb-Diät, die die Menge an Kohlenhydraten lediglich einschränkt, wird in der AtkinsDiät vollständig auf Kohlenhydrate verzichtet.

\section{Religiöse Essensvorschriften}

Nicht zu vergessen sind religiöse Essensvorschriften (Verzicht auf Schweinefleisch im Islam, koschere Lebensmittel im Judentum, Fastenzeiten und -regeln usw.).

\section{Literatur}

1 Raimann C, Ganz C, Garvelmann F Bertschi-Stahl H-D, Fehr-Streule R: Grundlagen der Traditionellen Europäischen $\mathrm{Na}$ turheilkunde. Schiedlberg, Bacopa, 2012.

2 Cybulka W: Die Heilmethode des Naturarztes Johann Schroth und seine ausgezeichneten Erfolge. Frankfurt/M., Bayrhoffer, 1846.

3 Pukownik P: Das Heilwissen der heiligen Hildegard von Bingen. Petersberg, Via Nova, 2011.

4 Wetter U: 5-Elemente-Küche. Aarau, AT, 1998.

5 D’Adamo PJ: 4 Blutgruppen, richtig leben. München, Piper, 2001.

6 Gittleman AL: Ernährung nach dem Stoffwechseltyp. Aitrang, Winpferd, 1998.

7 Coy J: Die neue Anti-Krebs-Ernährung. München, Gräfe und Unzer, 2009. 